\title{
Trastuzumab use in older patients with HER2-positive metastatic breast cancer: outcomes and treatment patterns in a whole-of-population Australian cohort (2003-2015)
}

Benjamin Daniels $^{1 *}$ (D), Belinda E. Kiely ${ }^{2}$, Monica Tang ${ }^{1,2}$, Hanna Tervonen ${ }^{1}$ and Sallie-Anne Pearson ${ }^{1}$

\begin{abstract}
Background: Older patients with HER2-positive metastatic breast (HER2 + MBC) cancer are underrepresented in clinical trials. We aim to describe the treatment patterns and overall survival (OS) for older women receiving trastuzumab for HER2 + MBC.

Methods: Retrospective, whole-of-population cohort study using demographic, dispensing, and medical services data for Australian women $\geq 65$ years initiating trastuzumab for HER2 + MBC between 2003 and 2015. We describe time-on-trastuzumab; type and timing of other cancer treatments; rates of cardiac monitoring; and OS from trastuzumab initiation for HER2 + MBC.

Results: Of 5404 women initiating trastuzumab for HER2 + MBC, 1583 (29\%) were $\geq 65$ years old, and the proportion of older patients increased from $20 \%$ in 2003 to 38\% in 2015. The median age for older women was 73 years and 516 (33\%) were $\geq 75$ years. Most older patients (92\%) received $\geq 3$ medicines for comorbidities other than cancer. Median (IQR) time on trastuzumab was 14.1 months (5.9-32.1) and on all chemotherapy was 5.6 months (3.3-10.8). $74 \%$ received $\geq 1$ chemotherapy agent and $56 \%$ received endocrine therapy. Half (49\%) of patients had a cardiac assessment prior to initiating trastuzumab and overall $1228(76 \%)$ had $\geq 1$ cardiac assessment during the study period. At a median follow-up of 6 years, $73 \%$ of patients had died and the median OS was 25.6 months (IQR 10.7-58.7).

Conclusions: Older patients comprise a growing proportion of patients treated with HER2-targeted therapies in the real-world but they remain underrepresented in trials of these agents. Few trials report duration or OS estimates for older patients but our estimates are similar to those from trials that have. Although cardiac monitoring was a requirement of accessing trastuzumab during our study period, many patients did not undergo a cardiac assessment.
\end{abstract}

Keywords: HER2-positive, Metastatic breast cancer, Trastuzumab, HER2-targeted therapy, Older, Elderly, Populationbased

\footnotetext{
* Correspondence: b.daniels@unsw.edu.au

${ }^{1}$ Medicines Policy Research Unit, Centre for Big Data Research in Health,

University of New South Wales (UNSW), Lowy Cancer Research Centre,

Kensington, NSW 2052, Australia

Full list of author information is available at the end of the article
}

(c) The Author(s). 2019 Open Access This article is distributed under the terms of the Creative Commons Attribution 4.0 International License (http://creativecommons.org/licenses/by/4.0/), which permits unrestricted use, distribution, and reproduction in any medium, provided you give appropriate credit to the original author(s) and the source, provide a link to the Creative Commons license, and indicate if changes were made. The Creative Commons Public Domain Dedication waiver (http://creativecommons.org/publicdomain/zero/1.0/) applies to the data made available in this article, unless otherwise stated. 


\section{Background}

Evidence generated in randomized controlled trials (RCT) about the benefits and risks of new breast cancer treatments forms the basis of treatment guidelines, regulatory decisions, and communications between clinicians and patients about their likely prognosis and experience while undergoing treatment. Because RCTs are conducted in selected patient populations under standardized conditions, trial efficacy and safety outcomes may not directly apply to the more heterogeneous populations treated in routine clinical practice $[1,2]$. Notably, trials seldom include older participants in proportions representative of the real-world clinical population. For example, $43 \%$ of newly diagnosed breast cancers occur in people over 65 years of age [3-5], but the proportion of participants $\geq 65$ in breast cancer clinical trials has been estimated at just 9\% [6]. Evidence around the efficacy and safety of new treatments is, therefore, largely absent for a substantial proportion of patients undergoing breast cancer treatment.

In the case of HER2-positive metastatic breast cancer $(\mathrm{HER} 2+\mathrm{MBC})$, treatment patterns and survival outcomes are not well understood in older patients. Pivotal trials of HER2-targeted agents indicate that survival estimates have been increasing over time [7-9], however, the trend in survival over time for older patients is unknown $[9,10]$, with most RCTs including only a small number of participants $\geq 65$. For example, in recent HER $2+$ MBC trials, approximately $15 \%$ of participants were $\geq 65$ and only $2-3 \%$ were $\geq 75$ [11-13]. In the original pivotal trial, which evaluated trastuzumab (Herceptin, Genentech, South San Francisco, CA; Hoffmann-La Roche Ltd., Basel, Switzerland) plus chemotherapy against chemotherapy alone, 23\% of patients were $>60$ and the oldest participant in the trial was $77[7,10]$. Sub-group analysis of these patients found that they received benefit in terms of overall survival (OS) from the addition of trastuzumab to chemotherapy, but rates of cardiotoxicity were double those reported in patients $\leq 60$ [10].

More recently, the CLEOPATRA trial, evaluating firstline pertuzumab (Perjeta, Genentech, South San Francisco, Ca; Hoffmann-La Roche Ltd., Basel, Switzerland), trastuzumab, and chemotherapy against trastuzumab plus chemotherapy, found the addition of pertuzumab was associated with increased progression-free survival (PFS) and OS in patients $\geq 65$ (16\% trial participants). However, there were no differences between treatment arms for patients $\geq 75$ ( $2 \%$ of trial participants) $[8,9]$. The incidence of cardiotoxic events was low and similar between older and younger patients in the CLEOPATRA trial [9].

The majority of observational research in older patients with HER2+ breast cancer has focused on earlystage disease [14]. There are few data from observational studies on the treatment and outcomes for older patients with HER $2+M B C$ and in the present study we sought to describe the real-world treatment patterns and survival outcomes for patients $\geq 65$ starting trastuzumab for HER2 + MBC.. Our specific objectives were to determine the: time-on-trastuzumab and other HER2-targeted therapies; time-on-other cancer therapies; patterns of cardiac monitoring; and OS in this older population.

\section{Methods}

\section{Setting and data}

The Australian healthcare system and the datasets we used in our study have been described previously in our research protocol [15]. Briefly, Australia maintains a publicly funded, universal healthcare system entitling all citizens and permanent residents to subsidized medicines through the Pharmaceutical Benefits Scheme (PBS) and subsidized medical services through the Medicare Benefits Schedule (MBS). The Herceptin Program, separate to the PBS, provided fully subsidized access to trastuzumab for HER2 + MBC from December 2001 until July 2015, when the program was closed and trastuzumab for HER2 + MBC was listed for subsidy on the PBS [15]. Lapatinib was subsidized by the PBS from May 2008 while pertuzumab and trastuzumab emtansine (T-DM1; Kadcyla, Genentech, South San Francisco, Ca; Hoffmann-La Roche Ltd., Basel, Switzerland) were subsidised from July 2015 [15].

The Australian Department of Human Services (DHS) - administering body for the Herceptin Program and PBS- supplied de-identified, patient-level data including: patient demographic information, HER2 testing results (immunohistochemistry (IHC) or in-situ hybridization (ISH)), records of trastuzumab dispensed to Herceptin Program enrollees, and all PBS dispensing records for Herceptin Program enrollees [15]. The DHS also provided the dispensing records for all patients in Australia accessing publicly-subsidized trastuzumab for early breast cancer (EBC) from 1 October 2006 to 30 June 2016. We determined previous treatment with trastuzumab for EBC through data linkage of Herceptin Program records with the dispensing records of patients who received trastuzumab for EBC.

The period of time observed across the datasets is 1 January 2001 to 30 June 2016.

\section{Study design and participants}

Our population-based, retrospective cohort study includes every Australian woman initiating trastuzumab for $\mathrm{MBC}$ subsidized through the Herceptin Program between 1 January 2003 and 30 June 2015. We considered older patients to be those initiating trastuzumab at age 65 or older. Our data collection is limited by a lack of clinico-pathological information and thus we have chosen not to make direct comparisons between older 
and younger populations in our study as we could not adjust for important confounders. However, we provided outcome estimates for patients $<65$ for descriptive context. All patients were observed from initiation of trastuzumab for MBC until death or 30 June 2016.

\section{Outcomes and statistical analysis}

We used descriptive statistics to summarize age, weight, the number and proportion of patients who died, and determination of HER2 + status (IHC or ISH). We used a validated algorithm, applied to the dispensing records from up to 1 year prior to initiation of trastuzumab for MBC for older patients, to identify treatment for comorbid disease [16]. PBS dispensing records contain all dispensed medicines for patients $\geq 65$ years and these records were used to estimate patient comorbidities. Patients $<65$ are required to pay a co-payment towards prescribed medications which ranged from \$23.00 AUD in 2003 to $\$ 37.70$ AUD in 2015. As PBS data are collected for the primary purpose of providing financial reimbursement from the federal government, medicines costing below this co-payment amount do not attract government reimbursement and, therefore, do not appear in PBS dispensing records because the patient bears the full cost of the medicine [17]. For this reason, we cannot be sure we observe all of the medicines dispensed to patients $<65$ and we restricted our estimates of comorbid treatments to patients $\geq 65$.

We estimated: OS from the time of first trastuzumab dispensing for HER2 + MBC until month of death (set at the last day of the month) or censor and; time on treatment as the period of time from first observed dispensing date of each therapy until the sooner of either the last observed dispensing date plus 30 days or the number of days to death $[18,19]$. We considered a period of $>$ 90 days between dispensings as a break in a course of treatment and a subsequent dispensing following a break of $>90$ days as beginning a new course of therapy [20].

We used PBS dispensing data to summarize the type, number, and timing of other cancer treatments dispensed following trastuzumab initiation for HER2 + $\mathrm{MBC}$. We determined first-line partner therapy based on treatments dispensed during the period from 30 days prior to 90 days following trastuzumab initiation. We calculated the time on other cancer therapies in the same manner as for HER2-targeted therapies, and summarized the number of unique chemotherapies dispensed following trastuzumab initiation.

For each patient we used MBS claims to identify the number of cardiac assessments (echocardiography and gated cardiac blood pool scans) and when they were performed. We defined baseline cardiac assessments as those assessments with a claim date from 60 days prior, to 30 days following the date of first dispensing of trastuzumab. We defined on-treatment cardiac assessments as those claimed from 30 days after the first dispensing until the end of the trastuzumab course [18].

All analyses were performed in SAS version 9.4 (SAS Institute, Cary, NC) with figures generated in R version 3.3.5.

\section{Results}

\section{Patient characteristics}

Of the 5404 women initiating trastuzumab for HER2 + MBC between 3 December 2001 and 30 June 2015, 1583 $(29 \%)$ were $\geq 65$. The median age for this older cohort was 73 years (IQR: $68-78)$, with $516(10 \%) \geq 75$ and 130 $(2 \%) \geq 85$. A minority of older women $(189 ; 12 \%)$ had received adjuvant trastuzumab. Most (1458; 92\%) had received three or more medicines to treat comorbid conditions prior to initiating trastuzumab (Table 1). The proportion of older patients initiating trastuzumab for MBC increased steadily over the study period, from $20 \%$ of all patients initiating trastuzumab for MBC in 2003 to 38\% in 2015 (Fig. 1, Additional file 1: Figure S1B). The median follow-up time for older patients was 5.7 years (IQR: 3.3-8.8).

\section{HER2-targeted therapy}

Median time on trastuzumab for HER $+\mathrm{MBC}$, excluding breaks, was 14.1 months (IQR: 5.9-31.1; Table 2) and the median duration of the first course of trastuzumab was 12.1 months (IQR: 4.6-24.9; Table 2). More than half of older patients $(838 ; 53 \%)$ continued trastuzumab beyond 1 year and 82 patients (5\%) continuously received the medicine for more than 5 years. Trastuzumab was started as monotherapy for $23 \%$ of older patients (Table 3), and trastuzumab was the only cancer therapy dispensed to $10 \%$ of older patients (Table 2).

Other HER2-targeted therapies were dispensed to 340 older patients (21\%): 91 (6\%) received pertuzumab, 182 (11\%) received lapatinib, and $101(6 \%)$ received T-DM1 (Table 2). The median time on all HER2-targeted therapies, including trastuzumab, was 15.4 months (IQR: 6.3-37.0) and the median proportion of survival time spent on HER2-targeted therapies was 85\% (IQR: 58-100\%; Table 2).

\section{Chemotherapy and endocrine therapy}

Chemotherapy was dispensed to 1170 (74\%) older patients and most of these patients received just one chemotherapy medicine (the median number of chemotherapies was 1 (IQR: 0-2; Table 2). The median time on all chemotherapy, excluding breaks in treatment, was 5.6 months (IQR: $3.3-10.8$ ). Most older patients received just one course of chemotherapy (67\%), while $13 \%$ received three or more courses of chemotherapy. Just over half (58\%) of older patients initiated trastuzumab with a chemotherapy partner, most commonly a taxane (50\%), and the proportion of patients initiating trastuzumab 
Table 1 Characteristics of patients 65 years and older, those younger than 65 years, and the entire cohort

\begin{tabular}{|c|c|c|c|}
\hline & $\begin{array}{l}\text { Older patients }(\geq \\
65)\end{array}$ & $\begin{array}{l}\text { Younger patients }(< \\
65)\end{array}$ & $\begin{array}{l}\text { All } \\
\text { patients }\end{array}$ \\
\hline Patients (n) & 1583 & 3821 & 5404 \\
\hline \multicolumn{4}{|l|}{ Baseline measures } \\
\hline Age in years at first metastatic trastuzumab dispensing, median (IQR) & $73(68-78)$ & $52(45-58)$ & $57(48-66)$ \\
\hline$<35$ & - & $138(4 \%)$ & $138(3 \%)$ \\
\hline $35-44$ & - & $760(20 \%)$ & $760(14 \%)$ \\
\hline $45-54$ & - & $1372(36 \%)$ & $\begin{array}{l}1372 \\
(25 \%)\end{array}$ \\
\hline $55-64$ & - & $1551(40 \%)$ & $\begin{array}{l}1551 \\
(29 \%)\end{array}$ \\
\hline $65-74$ & $937(59 \%)$ & & $937(17 \%)$ \\
\hline $75-84$ & $516(33 \%)$ & - & $516(10 \%)$ \\
\hline $85+$ & $130(8 \%)$ & - & $130(2 \%)$ \\
\hline Weight (kgs) at Herceptin Program enrolment, median (IQR) & $68(59-77)$ & $70(60-82)$ & $70(60-80)$ \\
\hline \multicolumn{4}{|l|}{ Treatment qualification: } \\
\hline HER2-positive by $\mathrm{IHC}^{\mathrm{a}, \mathrm{c}} 3+, \mathrm{n}$ & $871(55 \%)$ & $2285(60 \%)$ & $\begin{array}{l}3156 \\
(58 \%)\end{array}$ \\
\hline HER2-positive by ISH ${ }^{b, c}, n$ & $734(46 \%)$ & $1615(42 \%)$ & $\begin{array}{l}2349 \\
(43 \%)\end{array}$ \\
\hline Previously treated with trastuzumab for $\mathrm{EBC}, \mathrm{n}$ & $189(12 \%)$ & $612(16 \%)$ & $801(15 \%)$ \\
\hline \multicolumn{4}{|l|}{ Number of medicines dispensed to treat comorbidity, $n$ patients: ${ }^{d}$} \\
\hline No medicines & $52(3 \%)$ & - & - \\
\hline $1-2$ medicines & $73(5 \%)$ & - & - \\
\hline 3 or more medicines & $1458(92 \%)$ & - & - \\
\hline $\begin{array}{l}\text { Treatment with at least one cardiovascular medicine in the year prior to trastuzumab } \\
\text { initiatione }{ }^{e}, \mathrm{n}\end{array}$ & $1047(66 \%)$ & - & - \\
\hline \multicolumn{4}{|l|}{ Post-trastuzumab initiation measures } \\
\hline Deaths, n & $1153(73 \%)$ & $2622(69 \%)$ & $\begin{array}{l}3775 \\
(70 \%)\end{array}$ \\
\hline Median follow-up time ${ }^{f}$, years (IQR) & $5.7(3.3-8.8)$ & $6.7(3.9-10.3)$ & $\begin{array}{l}6.4(3.8- \\
9.9)\end{array}$ \\
\hline
\end{tabular}

${ }^{\mathrm{a}}$ Immunohistochemistry

${ }^{b}$ In-situ hybridisation

'Patients may have multiple tests

${ }^{\mathrm{d}}$ Number of comorbidities assessed using the RxRisk algorithm. For patients younger than 65 years of age, medicines costing below the PBS co-payment threshold are not captured in the data and a comorbidity estimate cannot be determined for these patients

"Cardiovascular medicines are those whose ATC code begins with " $\mathrm{C}$ "

${ }^{\mathrm{f}}$ Median follow-up time calculated according to the reverse Kaplan-Meier method

with chemotherapy increased slightly over the study period (Additional file 1: Figure S1C). Endocrine therapy was dispensed to 885 older patients (56\%) for a median duration of 14.5 months (IQR: 3.8-41.2; Table 2). One third of these patients initiated trastuzumab with an endocrine therapy alone ( $18 \%$ of all older patients) while the remaining patients started endocrine therapy after trastuzumab initiation.

\section{Cardiac monitoring}

At least one cardiac monitoring test was undertaken in 1228 (76\%) older patients: $782(49 \%)$ had a baseline test (60 days prior to 30 days after starting trastuzumab for
HER2+ MBC), 956 (60\%) had a test while receiving trastuzumab; 607 (38\%) had a test in both time periods (Table 3).

\section{Overall survival}

By 30 June 2016, 73\% of older patients had died. Median OS from initiation of trastuzumab for $\mathrm{MBC}$ was 25.6 months (IQR 10.7-58.7; Fig. 2). The median survival for older patients increased with each year of initiation between 2003 and 2015 (Fig. 3), but the increasing trend in median survival was limited to patients who were between ages 65 and 74 at initiation. Median survival for patients $\geq 75$ at initiation did not increase over the study period (Additional file 1: Figure S1A). Median survival at 


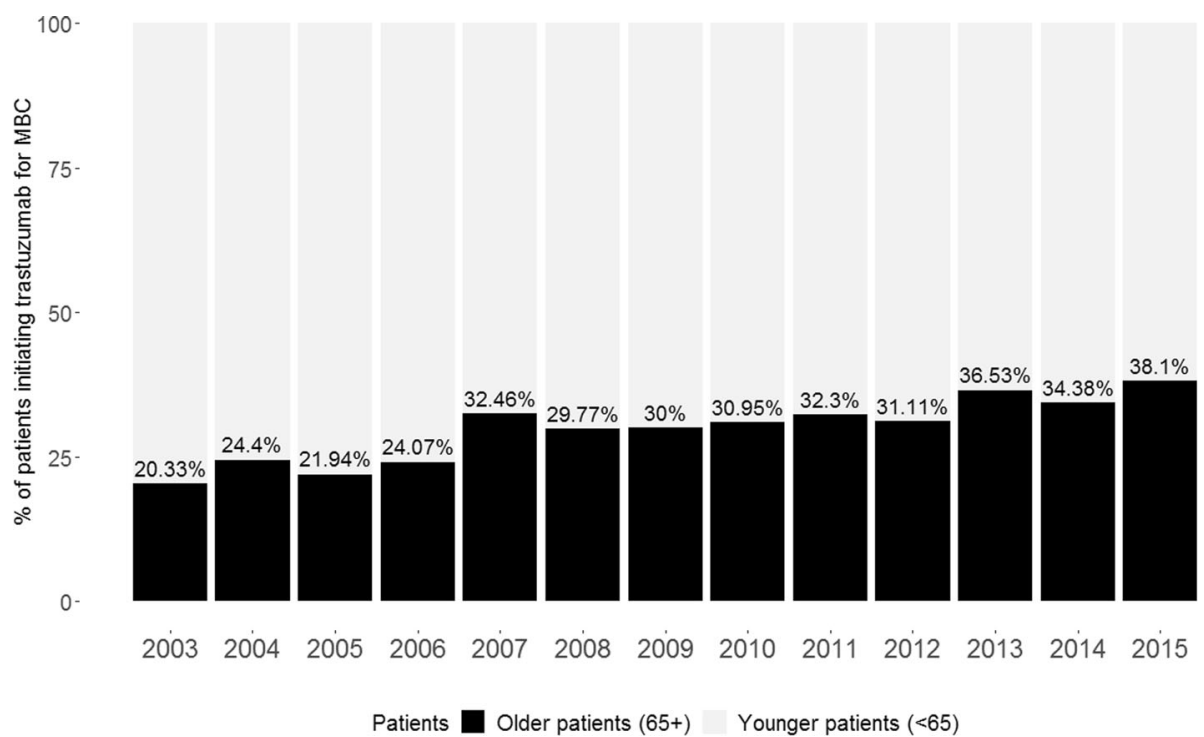

Fig. 1 Proportion of older patients initiating trastuzumab for MBC in each year

initiation was 29.9 months (IQR: 13.3-69.4) for patients aged 65-74 and 19.6 months (IQR: 7.4-47.4) for patients $\geq 75$ at initiation (Additional file 1: Figure S1D).

\section{Discussion}

In this population-based cohort study we described the largest cohort of older patients with HER2 + MBC, treated with trastuzumab, in the literature to date. There remains a lack of data about the treatment and outcomes experienced by older patients with HER2 + MBC and our study provides some clarity for these patients. We found that almost a third of all women initiating trastuzumab for MBC between 2003 and 2015 were $\geq 65$, with nearly as many women $\geq 75$ initiating treatment as those aged 35 to 44 . These proportions are substantially larger than those reported in clinical trials, where around $15 \%$ of patients were $\geq 65$ and less than $2 \%$ of patients were $\geq 75[9,12]$. Moreover, we found that the proportion of older patients treated with trastuzumab for MBC had roughly doubled between 2003 and 2015-from 20 to 38\%-but the median age of trial participants has only increased slightly (51 to 54 years) between the original pivotal trial of trastuzumab for MBC and the more recent CLEOPATRA and MARIANNE trials $[7,11,12]$. These findings highlight that older patients are still underrepresented in clinical trials of HER2-targeted agents.

The median duration of the first course of trastuzumab for older patients (12.1 months) was similar to median estimates reported for patients $\geq 65$ in the control arms of the CLEOPATRA and MARIANNE trials (trastuzumab plus taxane; 10.4 and 14.6 months, respectively)
$[9,12]$. We also observed a similar proportion of older patients with previous (neo) adjuvant trastuzumab therapy $(12 \%)$ as reported from the CLEOPATRA trial (11\%) [9], however, (neo) adjuvant trastuzumab was not publicly subsidised in Australia until October 2006 [15], and many patients in our cohort would not have had access to that treatment. The most recent HER2-targeted therapies-pertuzumab and T-DM1-only became subsidized in Australia from July 2015, so few older patients in our study received either medicine.

Survival estimates for patients $\geq 65$ in the CLEOPATRA and MARIANNE trials were not reported, however, subgroup analysis of patients $>60$ years from the original pivotal trial reported a median OS of 24 months, which is similar to our estimate of 25.6 months. We observed that survival estimates for older patients increased slightly over the study period, primarily in patients initiating trastuzumab for MBC between the ages of 65-74 (Additional file 1: Figure S1A). A similar finding has been reported from studies in wider MBC populations irrespective of HER2status [21-23], but not specifically in HER2 + MBC cohorts. Our survival estimates for older patients also indicated that nearly one quarter of older patients would survive at least 5 years from trastuzumab initiation for MBC. We have previously reported a similar rate of 5-year survival for trastuzumab-treated HER2 + MBC patients of all ages [19].

There are few observational studies examining the treatment and outcomes of older patients with HER2 + MBC. A handful of studies utilizing hospital-based cohorts have reported proportions as high as 28 and $14 \%$ for patients $\geq$ 
Table 2 Trastuzumab, HER2-targeted therapy, chemotherapy, and endocrine therapy treatments and durations

\begin{tabular}{|c|c|c|c|}
\hline & $\begin{array}{l}\text { Older patients } \\
(>65)\end{array}$ & $\begin{array}{l}\text { Younger patients } \\
(<65)\end{array}$ & All Patients \\
\hline Patients, n & 1583 & 3821 & 5404 \\
\hline \multicolumn{4}{|l|}{ Trastuzumab } \\
\hline \multicolumn{4}{|l|}{ Number of trastuzumab courses, ${ }^{a} n$ patients } \\
\hline One course & $1583(100 \%)$ & $3821(100 \%)$ & $\begin{array}{l}5404 \\
(100 \%)\end{array}$ \\
\hline Two courses & $319(20 \%)$ & $1079(28 \%)$ & $1398(26 \%)$ \\
\hline Three or more courses & $61(4 \%)$ & $341(9 \%)$ & $402(7 \%)$ \\
\hline Median time on all trastuzumab treatment, ${ }^{\mathrm{b}}$ months (IQR) & $14.1(5.9-32.1)$ & $18.2(8.7-40.9)$ & $\begin{array}{l}16.8(7.8- \\
38.2)\end{array}$ \\
\hline Median duration of the first uninterrupted course of trastuzumab, months (IQR) & $12.1(4.6-24.9)$ & $14.0(6.5-28.9)$ & $\begin{array}{l}13.5(5.9- \\
27.6)\end{array}$ \\
\hline Trastuzumab monotherapy only, $\mathrm{n}$ & $154(10 \%)$ & $163(4 \%)$ & $317(6 \%)$ \\
\hline \multicolumn{4}{|l|}{ HER2-targeted therapies } \\
\hline Treated with other HER2-targeted therapies for MBC, n: & $340(21 \%)$ & $1404(37 \%)$ & $1744(32 \%)$ \\
\hline Lapatinib & $182(11 \%)$ & $950(25 \%)$ & $1132(21 \%)$ \\
\hline Pertuzumab & $91(6 \%)$ & $284(7 \%)$ & $375(7 \%)$ \\
\hline T-DM1 & $101(6 \%)$ & $300(8 \%)$ & $401(7 \%)$ \\
\hline Median time on all HER2-targeted therapies, ${ }^{\mathrm{b}}$ months (IQR) & $15.4(6.3-37.0)$ & $21.5(10.1-48.8)$ & $\begin{array}{l}19.4(8.8- \\
45.0)\end{array}$ \\
\hline $\begin{array}{l}\text { Median proportions of observed survival time }{ }^{c} \text { on all HER2-targeted therapies, including } \\
\text { trastuzumab, \% (IQR) }\end{array}$ & $85 \%(58-100 \%)$ & $85 \%(61-99 \%)$ & $\begin{array}{l}85 \%(60- \\
99 \%)\end{array}$ \\
\hline \multicolumn{4}{|l|}{ Chemotherapy } \\
\hline Dispensed chemotherapy, n & $1170(74 \%)$ & $3342(87 \%)$ & $4512(83 \%)$ \\
\hline Median number of unique chemotherapies dispensed per person, n (IQR) & $1(0-2)$ & $2(1-3)$ & $2(1-3)$ \\
\hline Median time on all chemotherapy, ${ }^{b}$ months (IQR) & $5.6(3.3-10.8)$ & $8.3(4.5-16.5)$ & $\begin{array}{l}7.4(4.2- \\
15.1)\end{array}$ \\
\hline Median proportion of survival time on chemotherapy, \% (IQR) & $30 \%(13-53 \%)$ & $38 \%(18-62 \%)$ & $\begin{array}{l}36 \%(16- \\
60 \%)\end{array}$ \\
\hline \multicolumn{4}{|l|}{ Endocrine therapy } \\
\hline Dispensed endocrine therapy, $\mathrm{n}^{\mathrm{d}}$ & $885(56 \%)$ & $2179(57 \%)$ & $3064(57 \%)$ \\
\hline Median time on all endocrine therapy, months (IQR) & $14.5(3.8-41.2)$ & $16.4(4.9-43.6)$ & $\begin{array}{l}15.8(4.6- \\
42.6)\end{array}$ \\
\hline Median proportion of survival time on endocrine therapy, \% (IQR) & $60 \%(27-88 \%)$ & $49 \%(20-80 \%)$ & $\begin{array}{l}52 \%(21- \\
82 \%)\end{array}$ \\
\hline
\end{tabular}

${ }^{a}$ A course is defined as consecutive dispensings of trastuzumab closer in time than 90 days. A gap of $\geq 90$ days between trastuzumab dispensings was considered a break in trastuzumab therapy and the subsequent dispensing considered the start of a new course of therapy.

${ }^{b}$ Excluding breaks in treatment $\geq 90$ days

'Observed duration of treatment, excluding breaks, as a proportion of observed survival time from initiation of trastuzumab for MBC

${ }^{\mathrm{d}}$ Only endocrine therapies dispensed between 30 days prior to initiating trastuzumab for MBC and death or censor

65 and $\geq 75$ years, respectively [24, 25]. These studies have found that patients $\geq 65$ years received less chemotherapy compared to younger patients; had lower median OS; had higher underlying cardiovascular conditions at treatment initiation; and experienced higher rates of cardiotoxic events compared to younger patients [24, 25]. Jackisch et al. also reported that the proportion of older patients in their cohort of women with HER2+ MBC steadily increased during the 10 years of their study period [24].
Our study lacks detailed clinical data and we are unable to control for important patient and disease factors to allow robust, direct comparisons between the older and younger patients in our cohort. However, our findings align with those from previous studies in that we observed that a smaller proportion of older patients were dispensed chemotherapy, and a larger proportion of older patients received trastuzumab as monotherapy (no chemotherapy or endocrine therapy). Similar to Jackisch et al., we found that $18 \%$ of older patients and $13 \%$ of 
Table 3 First-line treatments and cardiac monitoring

\begin{tabular}{|c|c|c|c|}
\hline & Older patients $(\geq 65)$ & Younger patients $(<65)$ & Overall \\
\hline Patients, n & 1583 & 3821 & 5404 \\
\hline \multicolumn{4}{|l|}{ Number of patients initiating trastuzumab with ${ }^{a}$ : } \\
\hline Trastuzumab monotherapy & $372(23 \%)$ & $637(17 \%)$ & $1009(19 \%)$ \\
\hline Taxane and taxane combinations: & $787(50 \%)$ & $2368(62 \%)$ & $3155(58 \%)$ \\
\hline Endocrine therapy alone & $292(18 \%)$ & $490(13 \%)$ & $782(14 \%)$ \\
\hline Vinorelbine & $19(1 \%)$ & $60(2 \%)$ & $79(2 \%)$ \\
\hline Capecitabine & $40(3 \%)$ & $58(2 \%)$ & $98(2 \%)$ \\
\hline Other chemotherapy combinations & $73(5 \%)$ & $208(5 \%)$ & $281(5 \%)$ \\
\hline Patients with a baseline cardiac assessment, $n$ & $782(49 \%)$ & $1830(48 \%)$ & $2612(48 \%)$ \\
\hline Patients with a cardiac assessment while on trastuzumab, $n$ & $956(60 \%)$ & $2550(67 \%)$ & $3506(65 \%)$ \\
\hline Patients with a cardiac assessment at baseline and while on trastuzumab, $\mathrm{n}$ & $607(38 \%)$ & $1519(40 \%)$ & $2216(39 \%)$ \\
\hline Number of cardiac assessments perpatient, median (IQR) & $4(2-7)$ & $5(2-8)$ & $4(2-8)$ \\
\hline
\end{tabular}

${ }^{\text {a } F i r s t-l i n e ~ p a r t n e r ~ t h e r a p y ~ i s ~ b a s e d ~ o n ~ t r e a t m e n t s ~ d i s p e n s e d ~ d u r i n g ~ t h e ~ p e r i o d ~ f r o m ~} 30$ days prior to 90 days following trastuzumab initiation

younger patients initiated trastuzumab with endocrine therapy alone.

Despite the known cardiac risks of trastuzumab treatment, and the fact that older patients are more likely to have comorbidities that increase their risk of cardiotoxicity $[10,25,26]$, we observed that only half of the older patients in our cohort received a baseline cardiac assessment (49\%), and just over half (60\%) received an assessment while on treatment with trastuzumab. MBS data do not include claims for cardiac monitoring tests administered to public hospital inpatients and patients paying for the test out of pocket and it is possible that we do not observe cardiac assessments for some patients. However, previous work has estimated that the MBS captures at least $80 \%$ of cardiac monitoring tests [27]. Cardiac assessment was a prerequisite for enrolment in the Herceptin Program [15] but this finding suggests that many older patients did not receive this care. The findings were similar in patients $<65$ in our cohort where $48 \%$ received a baseline cardiac assessment.

As mentioned previously, the primary limitation of our study is a lack of clinical and pathological details. Consequently, we were unable to examine important clinical issues such treatment beyond disease progression and

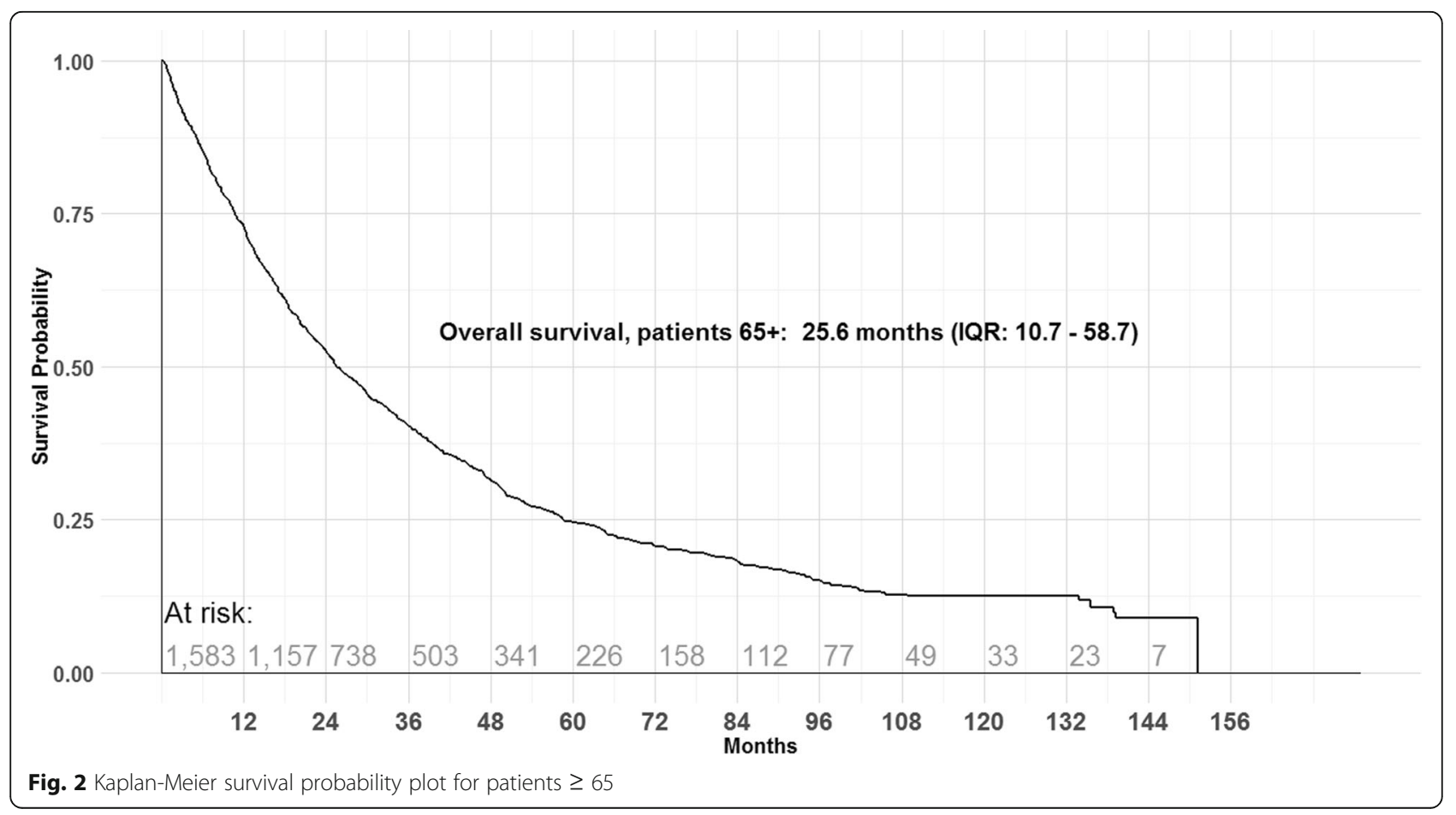




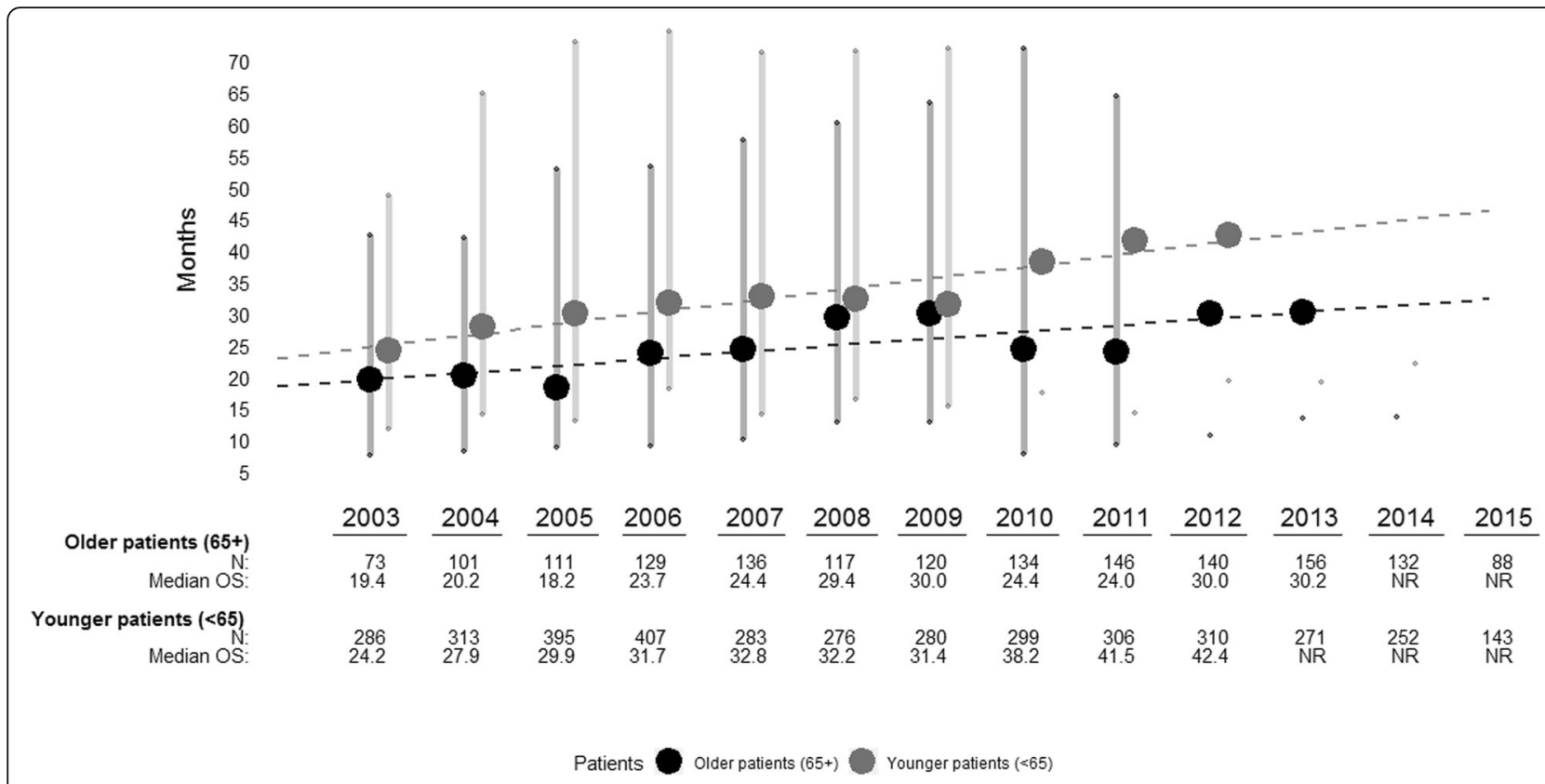

Fig. 3 Median overall survival (OS; large dots) and interquartile range (smaller dots and shaded bars) by year of trastuzumab for MBC initiation. The dotted lines indicate the trend in median OS over time. NR= median not reached

patient outcomes according to metastasis site, hormone receptor status, performance status, and comorbidities. The strengths of this study include the large size and the representativeness of sample drawn from the Australian population. The lengthy, 12.5 years of observation time allowed us to examine trends over time. Our cohort was selected from all women treated with publicly-funded trastuzumab for HER2 + MBC in Australia, which, given the high cost of trastuzumab, likely represents all Australian women treated during the study period. We do not have data for older women diagnosed with HER2 + $\mathrm{MBC}$ who did not receive trastuzumab and we cannot examine differences in survival or patient characterists between trastuzumab-treated and untreated HER2 + MBC patients. Furthermore, our data were collected for the primary purpose of reimbursement and they lack of clinical information. We are unable to describe the clinical and comorbidity characteristics of our older patients and how these factors might differ from younger patients initiating trastuzumab during the study period. We do not have diagnoses data and cannot determine which patients may have been diagnosed with early breast cancer; dates of disease progression; and dates of cardiac and other adverse events.

\section{Conclusions}

Our results provide generalizable estimates of time on treatment, OS, and the proportion of patients initiating HER2-targeted therapy from age 65. HER2 + MBC patients are living longer, and it is reassuring that approximately $25 \%$ of older patients starting trastuzumab for HER2+ MBC are living longer than 5 years. The survival gains from trastuzumab in older patients may not be as great as those in younger patients, possibly due to increased comorbidities and less administration of chemotherapy. Pertuzumab and T-DM1 can further improve survival outcomes without the toxicity of chemotherapy and these treatments may significantly impact on the survival of older patients. Future population cohort studies on the use of these newer agents in older patients will be useful. Our results highlight the fact that older patients are receiving HER2-targeted therapy in proportions larger than those seen in clinical trials and this proportion is growing. Policy makers will need to take this into account when making subsidy decisions for new targeted therapies.

\section{Supplementary information}

The online version of this article (https://doi.org/10.1186/s12885-019-6126-y) contains supplementary material, which is available to authorized users.

Additional file 1. Additional figures showing overall survival stratified by year of trastuzumab initiation and age group $(65-74,75+)$; proportion of patients initiating trastuzumab for $\mathrm{MBC}$ in each year, stratified by age group $(<65,65-74,75+)$; proportion of patients initiating trastuzumab with chemotherapy in each year, stratified by age group $(<65, \geq 65)$; and Kaplan-Meier survival probability plot for patients aged $65-74$ and patients aged $\geq 75$. (PDF $174 \mathrm{~kb}$ )

\section{Abbreviations}

AUD: Australian dollar; CLEOPATRA: Clinical Evaluation of Pertuzumab and Trastuzumab, phase III randomised controlled trial; DHS: Department of 
Human Services; EBC: Early breast cancer; HER2: Human epidermal growth factor 2 gene; HER2+MBC: Human epidermal growth factor 2 gene-positive metastatic breast cancer; IHC: Immunohistochemistry; IQR: Inter-quartile range; ISH: In-situ hybridisation; MARIANNE: A Randomized, 3 Arm, Multicenter, Phase III Study to Evaluate the Efficacy and the Safety of T-DM1 Combined With Pertuzumab or T-DM1 Combined With Pertuzumab-Placebo (Blinded for Pertuzumab), Versus the Combination of Trastuzumab Plus Taxane, as First Line Treatment in HER2 Positive Progressive or Recurrent Locally Advanced or Metastatic Breast Cancer; MBS: Medicare Benefits Schedule; MBC: Metastatic breast cancer; OS: Overall survival; PBS: Pharmaceutical Benefits Scheme; PFS: Progression-free survival; RCT: randomised controlled trial; T-DM1: Trastuzumab emtansine

\section{Acknowledgements}

We acknowledge the contribution of Sally Crossing (AM) (1946 - 2016) as the Health Consumer Advocate on this research program. We thank the Department of Human Services for providing the data for this research.

\section{Authors' contributions}

$B D, B E K, M T, H T$, and SAP significantly contributed to the study design. BD and SAP contributed to data acquisition, BD performed data analyses, and $B D, B E K, M T, H T$, and SAP interpreted the results. The manuscript was drafted by $B D$, and $B D, B E K, M T, H T$, and SAP revised the manuscript drafts critically and approved the final version of the manuscript.

\section{Funding}

This work was supported by the National Health and Medical Research Council Centre of Research Excellence in Medicines and Ageing (CREMA; ID: 1060407) and a Cooperative Research Centre Project (CRC-P) grant from the Australian Government Department of Industry, Innovation, and Science (ID: CRC-P-439). MT is supported by an NHMRC Postgraduate Research Scholarship (ID: 1151479), a National Breast Cancer Foundation Postgraduate Scholarship Top-Up (ID: DS-18-01), and a Translational Cancer Research Network Clinical PhD Scholarship Top-Up award, supported by the Cancer Institute NSW (no grant number). The funding bodies had no role in the design of the study, analysis of the data, interpretation of the findings, or the writing of the manuscript.

\section{Availability of data and materials}

Details of the source data and record linkage for this study are available in the study protocol [15]. Access to the datasets analysed for the current study is not permitted without the express permission of the approving Human Research Ethics Committee and the data custodian (DHS). Third parties can contact the DHS (https://www.humanservices.gov.au/,

statistics@humanservices.gov.au) regarding access to the source data and data custodian approval.

\section{Ethics approval and consent to participate}

Our (DHS) External Request Evaluation Committee (Approval Numbers: MI1474, MI1475, MI1477, study was approved by the NSW Population and Health Services Research Ethics Committee (Approval Number: 2010/02/213) and data access was granted by the Australian Department of Human Services (DHS) (MI5858). Individual consent for the release of these data has been waived according to the Australian Privacy Act of 1988 [15].

\section{Consent for publication}

Not applicable.

\section{Competing interests}

BEK has received conference support and speaker's honorariums from Roche and advisory board honorariums from Novartis and Teva. MT has received travel support from Roche. SAP is a member of the Drug Utilisation Sub Committee of the Pharmaceutical Benefits Advisory Committee. The views expressed in this paper do not represent those of the either committee. The remaining authors declare no conflicts of interest.

\section{Author details}

'Medicines Policy Research Unit, Centre for Big Data Research in Health, University of New South Wales (UNSW), Lowy Cancer Research Centre, Kensington, NSW 2052, Australia. ${ }^{2}$ NHMRC Clinical Trials Centre, University of Sydney, Sydney, Australia.
Received: 2 December 2018 Accepted: 3 September 2019 Published online: 11 September 2019

\section{References}

1. Rawlins M. De testimonio: on the evidence for decisions about the use of therapeutic interventions. Lancet. 2008;372(9656):2152-61.

2. van de Water W, Kiderlen M, Bastiaannet E, Siesling S, Westendorp RG, van de Velde CJ, et al. External validity of a trial comprised of elderly patients with hormone receptor-positive breast cancer. J Natl Cancer Inst. 2014; 106(4):dju051.

3. National Cancer Institute. Cancer Stat Facts: Cancer of Any Site Available from: https://seer.cancer.gov/statfacts/html/all.html. Accessed 27 Sept 2018.

4. National Cancer Institute. Cancer Stat Facts: Female Breast Cancer Available from: https://seer.cancer.gov/statfacts/html/breast.html. Accessed 27 Sept 2018.

5. Siegel RL, Miller KD, Jemal A. Cancer statistics, 2017. CA Cancer J Clin. 2017;67(1):7-30.

6. Hutchins LF, Unger JM, Crowley JJ, Coltman CA Jr, Albain KS. Underrepresentation of patients 65 years of age or older in cancer-treatment trials. N Engl J Med. 1999;341(27):2061-7.

7. Slamon DJ, Leyland-Jones B, Shak S, Fuchs H, Paton V, Bajamonde A, et al. Use of chemotherapy plus a monoclonal antibody against HER2 for metastatic breast cancer that overexpresses HER2. N Engl J Med. 2001; 344(11):783-92.

8. Swain SM, Baselga J, Kim SB, Ro J, Semiglazov V, Campone M, et al. Pertuzumab, trastuzumab, and docetaxel in HER2-positive metastatic breast cancer. N Engl J Med. 2015;372(8):724-34.

9. Miles D, Baselga J, Amadori D, Sunpaweravong P, Semiglazov V, Knott A, et al. Treatment of older patients with HER2-positive metastatic breast cancer with pertuzumab, trastuzumab, and docetaxel: subgroup analyses from a randomized, double-blind, placebo-controlled phase III trial (CLEOPATRA). Breast Cancer Res Treat. 2013;142(1):89-99.

10. Fyfe G, Mass R, Murphy M, Slamon D. Older (age > 60 years) patients obtain survival benefit from herceptin plus chemotherapy. Eur J Cancer. 2001;37:S189-S90.

11. Baselga J, Cortes J, Kim SB, Im SA, Hegg R, Im YH, et al. Pertuzumab plus trastuzumab plus docetaxel for metastatic breast cancer. N Engl J Med. 2012;366(2):109-19.

12. Perez EA, Barrios C, Eiermann W, Toi M, Im YH, Conte P, et al. Trastuzumab Emtansine with or without Pertuzumab versus Trastuzumab plus Taxane for human epidermal growth factor receptor 2-positive, advanced breast Cancer: primary results from the phase III MARIANNE study. J Clin Oncol. 2017:35(2):141-8.

13. Krop IE, Kim SB, Martin AG, LoRusso PM, Ferrero JM, Badovinac-Crnjevic T, et al. Trastuzumab emtansine versus treatment of physician's choice in patients with previously treated HER2-positive metastatic breast cancer (TH3RESA): final overall survival results from a randomised open-label phase 3 trial. Lancet Oncol. 2017;18(6):743-54.

14. Daste A, Chakiba C, Domblides C, Gross-goupil M, Quivy A, Ravaud A, et al. Targeted therapy and elderly people: a review. Eur J Cancer. 2016:69:199-215.

15. Daniels B, Lord SJ, Kiely BE, Houssami N, Haywood P, Lu CY, et al. Use and outcomes of targeted therapies in early and metastatic HER2-positive breast cancer in Australia: protocol detailing observations in a whole of population cohort. BMJ Open. 2017;7(1). https://bmjopen.bmj.com/content/7/1/e01443 9.citation-tools.

16. Lu CY, Barratt J, Vitry A, Roughead E. Charlson and Rx-risk comorbidity indices were predictive of mortality in the Australian health care setting. J Clin Epidemiol. 2011;64(2):223-8.

17. Mellish L, Karanges EA, Litchfield MJ, Schaffer AL, Blanch B, Daniels BJ, et al. The Australian pharmaceutical benefits scheme data collection: a practical guide for researchers. BMC Res Notes. 2015;8(1):634.

18. Daniels B, Kiely BE, Lord SJ, Houssami N, Lu CY, Ward RL, et al. Trastuzumab for metastatic breast cancer: Real world outcomes from an Australian whole-of-population cohort (2001-2016). Breast. 2017;38:7-13.

19. Daniels B, Kiely BE, Lord SJ, Houssami N, Lu CY, Ward RL, et al. Long-term survival in trastuzumab-treated patients with HER2-positive metastatic breast cancer: real-world outcomes and treatment patterns in a whole-of-population Australian cohort (2001-2016). Breast Cancer Res Treat. 2018;171:151-9.

20. Pearson SA, Ringland CL, Ward RL. Trastuzumab and metastatic breast cancer: trastuzumab use in Australia--monitoring the effect of an expensive medicine access program. J Clin Oncol. 2007;25(24):3688-93. 
21. Zeng C, Wen W, Morgans AK, Pao W, Shu XO, Zheng W. Disparities by race, age, and sex in the improvement of survival for major cancers: results from the National Cancer Institute surveillance, epidemiology, and end results (SEER) program in the United States, 1990 to 2010. JAMA Oncol. 2015;1(1):88-96.

22. Foukakis T, Fornander T, Lekberg T, Hellborg H, Adolfsson J, Bergh J. Agespecific trends of survival in metastatic breast cancer: 26 years longitudinal data from a population-based cancer registry in Stockholm, Sweden. Breast Cancer Res Treat. 2011;130(2):553-60.

23. Ruiterkamp J, Ernst MF, de Munck L, van der Heiden-van der Loo M, Bastiaannet $E$, van de Poll-Franse LV, et al. Improved survival of patients with primary distant metastatic breast cancer in the period of 1995-2008. A nationwide population-based study in the Netherlands. Breast Cancer Res Treat. 2011;128(2):495-503.

24. Jackisch C, Schoenegg W, Reichert DA, Welslau M, Selbach J, Harich H-D, et al. Long-term results on trastuzumab (T) in elderly patients (EP) with locally advanced or metastatic HER2-positive breast cancer (BC). J Clin Oncol. 2014; 32(15_suppl):624.

25. Kaufman PA, Brufsky AM, Mayer M, Rugo HS, Tripathy D, Yood MU, et al. Treatment patterns and clinical outcomes in elderly patients with HER2positive metastatic breast cancer from the registHER observational study. Breast Cancer Res Treat. 2012:135(3):875-83.

26. Denegri A, Moccetti T, Moccetti M, Spallarossa P, Brunelli C, Ameri P. Cardiac toxicity of trastuzumab in elderly patients with breast cancer. J Geriatr Cardiol. 2016;13(4):355-63.

27. Lu CY, Srasuebkul P, Drew AK, Ward RL, Pearson SA. Positive spillover effects of prescribing requirements: increased cardiac testing in patients treated with trastuzumab for HER2+ metastatic breast cancer. Intern Med J. 2012; 42(11):1229-35.

\section{Publisher's Note}

Springer Nature remains neutral with regard to jurisdictional claims in published maps and institutional affiliations.

Ready to submit your research? Choose BMC and benefit from:

- fast, convenient online submission

- thorough peer review by experienced researchers in your field

- rapid publication on acceptance

- support for research data, including large and complex data types

- gold Open Access which fosters wider collaboration and increased citations

- maximum visibility for your research: over $100 \mathrm{M}$ website views per year

At $\mathrm{BMC}$, research is always in progress.

Learn more biomedcentral.com/submissions 